\title{
COMPARISON OF MORPHOLOGICAL AND CHEMICAL CHARACTERISTICS OF TWO SELECTED ACCESSIONS AND SIX WILD SPECIES OF GENUS Cinnamomum SCHAEFF
}

Ariyarathne H.B.M.A. ${ }^{1}$, Weerasuriya S.N. ${ }^{2}$, Senarath W.T.P.S.K. ${ }^{*}$

${ }^{1}$ Department of Botany, Faculty of Applied Science, University of Sri Jayawardenepura, Gangodawila, Nugegoda, Sri Lanka.

${ }^{2}$ National Cinnamon Research and Training Center, Dept. of Export Agriculture, Palolpitiya, Thihagoda, Matara, Sri Lanka.

\begin{abstract}
Cinnamon is increasingly becoming an important and potential foreign exchange earner and it is the most important spice in Sri Lanka. However, currently only two selected cinnamon accessions are available in Sri Lanka. Therefore, establishment of more commercial cinnamon varieties/ cultivars with high quality and yield are required. Experiments were carried out to investigate and compare of morphological and chemical characteristics of two selected cinnamon accessions (Sri Wijaya and Sri Gemunu) and six wild cinnamon species (Cinnamomum citriodorum, Cinnamomum capparu coronde, Cinnamomum litseaefolium, Cinnamomum revulorum, Cinnamomum dubium and Cinnamomum sinharajanse). Morphological characters of flower, leaf, seed and bark, yield of bark and leaf oil, and chemical characters of leaf and bark oil were examined. Leaf morphological characters; leaf shape, type of venation, leaf apex shape, leaf length and width, petiole length and leaf area were varied among species studied. Bark color, surface texture of bark, bark odor and seed color also helped to differentiate studied species. Highest mean leaf oil percentage and bark oil percentage was obtained from $C$. zeylanicum (Sri Gemunu) and $C$. zeylanicum (Sri Wijaya) respectively. The lowest mean leaf oil percentage was obtained from $C$. citriodorum and the lowest mean bark oil percentage was obtained from $C$. dubium. GC-MS analysis indicated presence of many economically important compounds such as eugenol, cinnamaldehyde, benzyl benzoate etc., in leaf and bark oil of all studied species. Abundance of considered favorable characters which are already consisted with cultivated accessions Sri Gemunu and Sri Wijaya bark and leaf oil with important characters and easy peeling ability has been observed in tested wild types, indicating the possibilities of improvement of Ceylon cinnamon for these characters through hybridization programme using wild relatives.
\end{abstract}

Key words: cinnamon, wild species, cultivated accessions, GC-MS analysis, bark oil, leaf oil.

*Corresponding author: E-mail: wtpsk2011@sjp.ac.lk

https://orcid.org/0000-0001-9691-8112

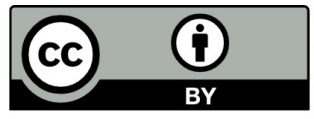

This is an open-access article distributed under the terms of the Creative Commons Attribution License, which permits unrestricted use, distribution and reproduction in any medium, provided the original author and source are credited. 


\section{Introduction}

Cinnamomum Schaeff. is one of the most important genus of family Lauraceae which consist of both crop and wild species. Nine different Cinnamomum species have been identified in Sri Lanka and out of them, $C$. zeylancium Blume. is an indigenous species while $C$. camphora (L) Presl. is an introduced species to Sri Lanka. The other seven (07) Cinnamomum species - C. capparucoronde Blume., C. citriodorum Thw., $C$. dubium Nees., $C$. litseaefolium Thw., $C$. ovalifolium Weight., $C$. rivulorum Kosterm. and $C$. sinharajanse Kosterm.grow in wild and endemic to Sri Lanka (Kostermans, 1995).

Genus Cinnamomum has high economic importance as $C$. zeylanicum, which is also considered as true cinnamon is globally demanded as a high priced spice. This is the oldest known perennial spice grown and traded in Sri Lanka (Saumyasiri et al., 2006). Dried bark of the stem in the form of quills and chips, leaf oil, bark oil and root oil of $C$. zeylanicum are the most demanding commercial products not only to use as a spice but also as a natural product or health food.

Studies on genus Cinnamomum are very rare, except for $C$. zeylanicum which is the cultivated species. Information on the current status of endemic wild cinnamon in Sri Lanka is limited (Saumyasiri et al., 2006) thus wild species are less recognized by the society. However, economically and taxonomically significant characteristics, such as erectness of stem, easy peeling ability, resistance to pest and diseases, presence of important secondary metabolites etc. may present in each species which is not yet been studied. Most of these wild cinnamon species have been used in indigenous medicine, thus has a high ethnobotanical importance as well.
Crop wild relatives are close relatives of cultivated plants and an essential component of natural and agricultural ecosystems (Groot et al., 2002). Determining the morphological and phytochemical characteristics of these wild endemics in comparison to cultivated and commercialized species is highly beneficial for the conservation, crop improvement programs and development of cinnamon industry in future.

Though Sri Lanka is the largest and best quality true cinnamon producer in the world, only two varieties namely Sri Gemunu and Sri Wijaya are cultivated (Wijesinghe et al., 2004). Thus it is indeed a need to identify quality and high yielding varieties from crop wild relatives for further development of cinnamon industry in Sri Lanka. On the other hand characterization of wild cinnamon species and comparison of those characteristics with cultivated species facilitate the conservation of wild species while providing a wide genetic base for crop improvement of commercial varieties (Sritharan, 1984). In the present study morphological, phytochemical and organoleptic characteristics of two selected cultivated cinnamon accessions and six wild cinnamon species, has been studied in order to identify variability and interspecific-relationships among them.

\section{Material and Methods}

C. zeylanicum (Sri Gemunu) $\left(\mathrm{C}_{1}\right)$ and $C$. zeylanicum (Sri Wijaya) $\left(\mathrm{C}_{2}\right)$ were selected as two cultivated accessions as those have high commercial importance. Six wild species $C$. citriodorum $\left(\mathrm{C}_{3}\right), C$. capparu coronde $\left(\mathrm{C}_{4}\right), C$. litseaefolium $\left(\mathrm{C}_{5}\right), C$. revulorum $\left(\mathrm{C}_{6}\right), C$. dubium $\left(\mathrm{C}_{7}\right)$ and $C$. sinharajanse $\left(\mathrm{C}_{8}\right)$ which were grown under same environmental conditions were also used in the present study as the morphology and phytochemical constituents could vary with the interaction of microclimatic conditions. Samples were collected from plants of the 
same maturity stage during the period of March to December 2015. Voucher specimens were prepared and identified using specimens at the National Herbarium at Royal Botanical Gardens, Sri Lanka. All data collected on characterization were analyzed using Statistical Analysis System (SAS), 9.3 version statistical package and Duncan Grouping was used whenever needed.

\subsection{Determination and comparison of morphological characters}

Mature leaves ( $5^{\text {th }}$ leaf from the tip of the branch with no symptoms of pest or diseases) were collected randomly from the first secondary branch of each study species in triplicates separately. There were ten leaves in each replicate, which counts to thirty leaves from each species. Leaf length (along the mid rib to obtain maximum length), leaf width and leaf weight were measured. Leaf area was measured using grid counting method. Petiole lengths of leaves were measured using a Vernier caliper. Colors of the leaves and flush were recorded using plant 'Munsell' color chart.

Freshly opened cinnamon flowers were collected, fixed and stored in $70 \%$ alcohol using method described by Kush and Stebbins (1961). Floral parts were observed under the dissecting microscope. Photographs and taxonomic illustrations of the flowers collected were made wherever possible. Mature seeds were also collected according to their availability during the study period. Seed length and seed width were measured using a Vernier caliper and the seed colors were also recorded. The color of the bark, peeling ability, surface texture and odor of bark were also recorded.

\subsection{Determination of leaf and bark oil yield}

Leaf samples were air dried for 4 to 5 days separately. Using Dean and Stark distillation method (Gursale et al., 2010) the moisture content of $5.0 \mathrm{~g}$ of each air dried leaf sample (Equation 1) and the dry matter content of the leaf samples taken for oil extraction (Equation 2) were determined.

Moisture percentage $=$ [Volume of water $(\mathrm{mL})$ / Weight of sample (g)] * 100 (1)

Dry matter content $=$ Weight of the leaf samples - Weight of moisture content in sample (2)

Modified Clevenger method (Gursale et al., 2010) and water distillation method (Gursale et al., 2010) were used to extract the leaf oil. Air dried leaf samples (50.0 g) from different test species were separately distilled over 4 hours. Extracted oil was weighed and the colour of the oil was noted. Volatile oil percentage (dry basis) was calculated for tested samples (Equation 3).

Volatile oil percentage $=[$ Weight of oil $(\mathrm{g})$ / Dry matter content of the sample (g)] * 100 (3)

To determine the bark oil content three separate bark samples (same maturity stage) were peeled off and air dried for 4 to 5 days separately. Then the oil content was determined using the same procedure used in leaf samples. However, in bark oil extraction a different graduated tube of the Clevenger arm filled with water was used and $3.0 \mathrm{ml}$ of the mixture of Hexane: Ether (2:1 solution) was added.

\subsection{Qualitative analysis of leaf and bark oil content using GC-MS}

Phytochemicals present in extracted leaf and bark oil were determined qualitatively and compared using Gas Chromatography - Mass Spectroscopy (GC-MS) (Shimadzu GC MS - QP2010). The sample was subjected to Agilent Technologies 7890A GC system coupled with (an Agilent) 5975C Mass Selective detector. A HP-5MS capillary column (30 $\mathrm{m} \times 0.25 \mathrm{~mm}$ internal diameter, $0.25 \mu \mathrm{m}$ film thickness) was used. The carrier gas was helium. The injector temperature was set at $320^{\circ} \mathrm{C}$. The initial oven temperature was at $70^{\circ} \mathrm{C}$ which was programmed to increase to $280^{\circ} \mathrm{C}$ at the rate of $10^{\circ} \mathrm{C} / \mathrm{min}$ with a hold time of $4 \mathrm{~min}$ 
at each increment. Injections of $1.0 \mu \mathrm{l}$ were made in split mode with a split ratio of 100:1. The mass spectrometer was operated in the electron ionization mode at $70 \mathrm{eV}$ and electron multiplier voltage at $1859 \mathrm{~V}$. Other MS operating parameters were as follows: ion source temperature $\quad 230^{\circ} \mathrm{C}, \quad$ quadrupole temperature $150^{\circ} \mathrm{C}$, solvent delay $3 \mathrm{~min}$ and scan range $33-550$ amu. GC-MS running time was set for $45 \mathrm{~min}$. The compounds were identified by direct comparison of the mass spectrum of the analyze at a particular retention time to that of a reference standard found in the National Institute of Standards and Technology (NIST) library. Chromatograms obtained were used in order to determine and compare the phytochemicals present in bark and leaf oil among the study species. At least $80 \%$ similarity index was considered significant (Kulathilaka and Senarath, 2013). Total GC-MS running time was 45 minutes.

\subsection{Quantification of selected economically} important phytochemicals present in leaf and bark oil

Three economically important phytochemicals (eugenol, cinnamaldehyde and benzyl benzoate) were selected for quantification purpose and analyzed using GC-MS. External standard method was used to compare peak areas with the amounts of standards injected. Quantification of eugenol, cinnamaldehyde and benzyl benzoate were carried out by using respective calibration curves of standards. A dendrogram was constructed based on oil yield characteristics, morphological and chemical characters using MINITAB (version 15) statistical package (Multivariate method of analysis).

\section{Results}

\subsection{Determination and comparison of} morphological characters

The leaves of Cinnamomum. species exhibited a wide range of variation in the morphological features such as shape, size, texture etc.

Among species used in the present study, $C$. sinharajanse showed the highest mean fresh weight of leaf with petiole. Lowest mean fresh weight of leaf with petiole was observed in $C$. citriodorum. There was no significant difference among $C$. zeylanicum (Sri Wijaya), $C$. capparu coronde, $C$. litseaefolium and $C$. dubium in mean fresh leaf weight. However, a significantly difference in fresh leaf weight was observed in C. zeylanicum (Sri Gemunu), C. citriodorum, C. revulorum and $C$. sinharajanse while no significant difference was observed between $C$. citriodorum and $C$. revulorum in mean fresh leaf weight (Table 1).

Further, mean fresh leaf weight of $C$. zeylanicum (Sri Gemunu) and $C$. sinharajanse were significantly higher than all the other tested species. Highest leaf lengths were observed in $C$. sinharajanse. Longer leaves were observed in $C$. dubium and were not significantly different from those of $\mathrm{C}$. sinharajanse. Mean leaf lengths of $C$. zeylanicum (Sri Wijaya), C. litseaefolium and $C$. revulorum were more or less similar while shortest leaves were observed in $C$ citriodorum.

A significantly higher mean leaf width was also observed in $C$. sinharajanse indicating that this has bigger leaves than all other tested species while $C$ citriodorum showed the smallest leaf width which is significantly lower than other species studied indicating this has the smallest leaf structure. The highest mean leaf area was observed from $C$. sinharajanse and the lowest mean leaf area was observed from $C$. citriodorum.

C. sinharajanse bears longest petioles while lowest length of petiole was obtained in $C$. citriodorum. However, mean petiole lengths of $C$. zeylanicum (Sri Wijaya) and $C$. revulorum were not significantly different. Mean leaf area of $C$. capparu coronde and C. zeylanicum (Sri gemunu) were not significantly different from each other. 
Except C. zeylanicum (Sri Gemunu), $C$. litseaefolium, and $C$. sinharajanse other studied species have lanceolate leaves. $C$. citriodorum and $C$. litseaefolium showed less dominant three vain structures while all other study species showed the prominent three vein distribution patterns. C. zeylanicum (Sri Gemunu) and C. sinharajanse showed ovate to eliptic leaf apices. $C$. litseaefolium and $C$. zeylanicum (Sri Wijaya) showed acute leaf apices and other study species showed acuminate leaf apices. All of the studied species showed obtuse leaf bases, entire leaf margins and opposite/sub opposite leaf arrangement. sinharajanse showed the flush colour of 5GY $7 / 10$ and $C$. capparu coronde, $C$. revulorum and $C$. dubium have entirely different flush colour (Table 2).

All the flowers found to be small and cream in colour with nine functional and three non-functional stamens (staminodes). Functional stamens are in two whorls, three in inner whorl and six in outer whorl. The ovary is superior monocarpellary and contains only one ovule. Placentation of the ovule is basal.

Table 1: Qualitative and Quantitative variation of leaf morphological characters

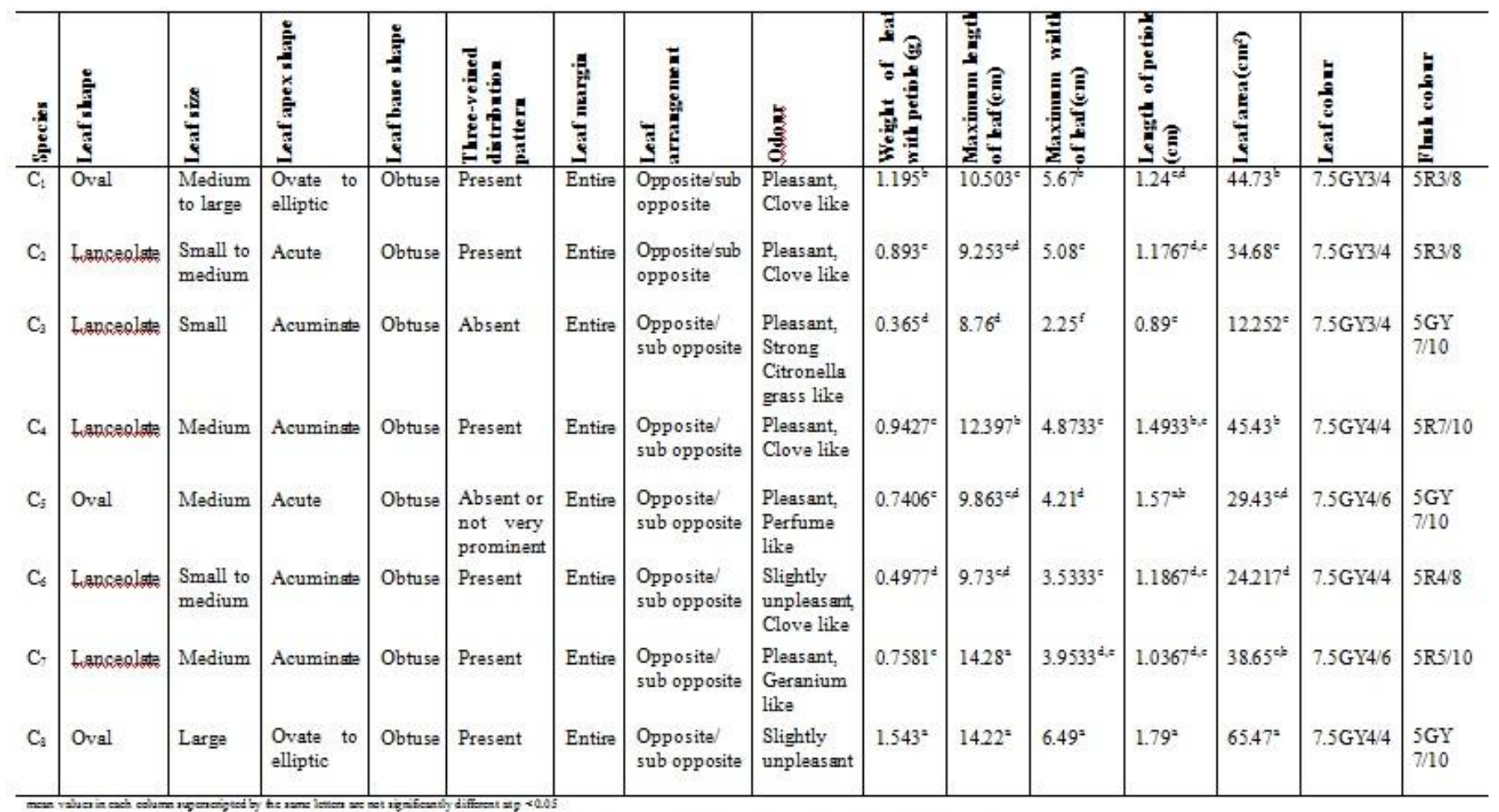

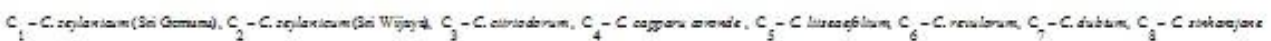

Leaf colour of all study species showed the 7.5 Greenish Yellow (GY) colour category of Plant Munsell colour chart (Figure 1). The flush colour of the two cultivated cinnamon accessions were different from all others and fall under 5R 3/8 of Plant Munsell colour chart while $C$. citriodorum, $C$. litseaefolium and $C$.
The highest seed length was obtained in C. sinharajanse $(1.19 \mathrm{~cm})$ and the highest seed width was obtained in $C$. zeylanicum (Sri Wijaya) $(0.73 \mathrm{~cm})$. When consider about the colour, $C$. zeylanicum (Sri Gemunu) and $C$. zeylanicum (Sri Wijaya) have same colour of the seeds (yellowish gray with brown streaks) which was 
Table 2: Variation of mean oil percentages and mean moisture percentages of leaf and bark

\begin{tabular}{|c|c|c|c|c|}
\hline \multirow[b]{2}{*}{ Species } & \multicolumn{2}{|l|}{ Leaf oil } & \multicolumn{2}{|l|}{ Bark oil } \\
\hline & $\begin{array}{l}\text { Mexn T.eaf } \\
\text { oil }(\%)\end{array}$ & $\begin{array}{l}\text { Mexn leaf } \\
\text { moisture }(\%)\end{array}$ & $\begin{array}{l}\text { Mean hark oil } \\
(\%)\end{array}$ & $\begin{array}{l}\text { Mean hark } \\
\text { moisture }(\%)\end{array}$ \\
\hline C.zevlanicum (Sri Gemunu) & $3.16^{\mathrm{a}}$ & 8.0 & $1.49^{2}$ & 17.0 \\
\hline C. zevlanicum (Sri Wijaya) & $2.96^{\circ}$ & 15.0 & $1.52^{\mathrm{a}}$ & 44.0 \\
\hline C citciodarum. & $06)^{f}$ & 240 & $078^{\circ}$ & 114 \\
\hline C. capparu caronder & $1.66^{b}$ & 15.0 & $0.48^{\circ}$ & 14.0 \\
\hline C. litsecalofium. & $0.99:$ & 14.0 & $0.77^{\circ}$ & 13.0 \\
\hline C. revularum & $0.39 s$ & 13.0 & $1.16^{\mathrm{b}}$ & 20.0 \\
\hline C. dubizum & $1.69^{d}$ & 13.0 & $0.41^{6}$ & 46.0 \\
\hline C. simharajanse & $2.64^{\circ}$ & 11.0 & $1.21^{b}$ & 1.6 .0 \\
\hline
\end{tabular}

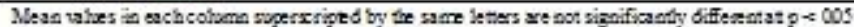
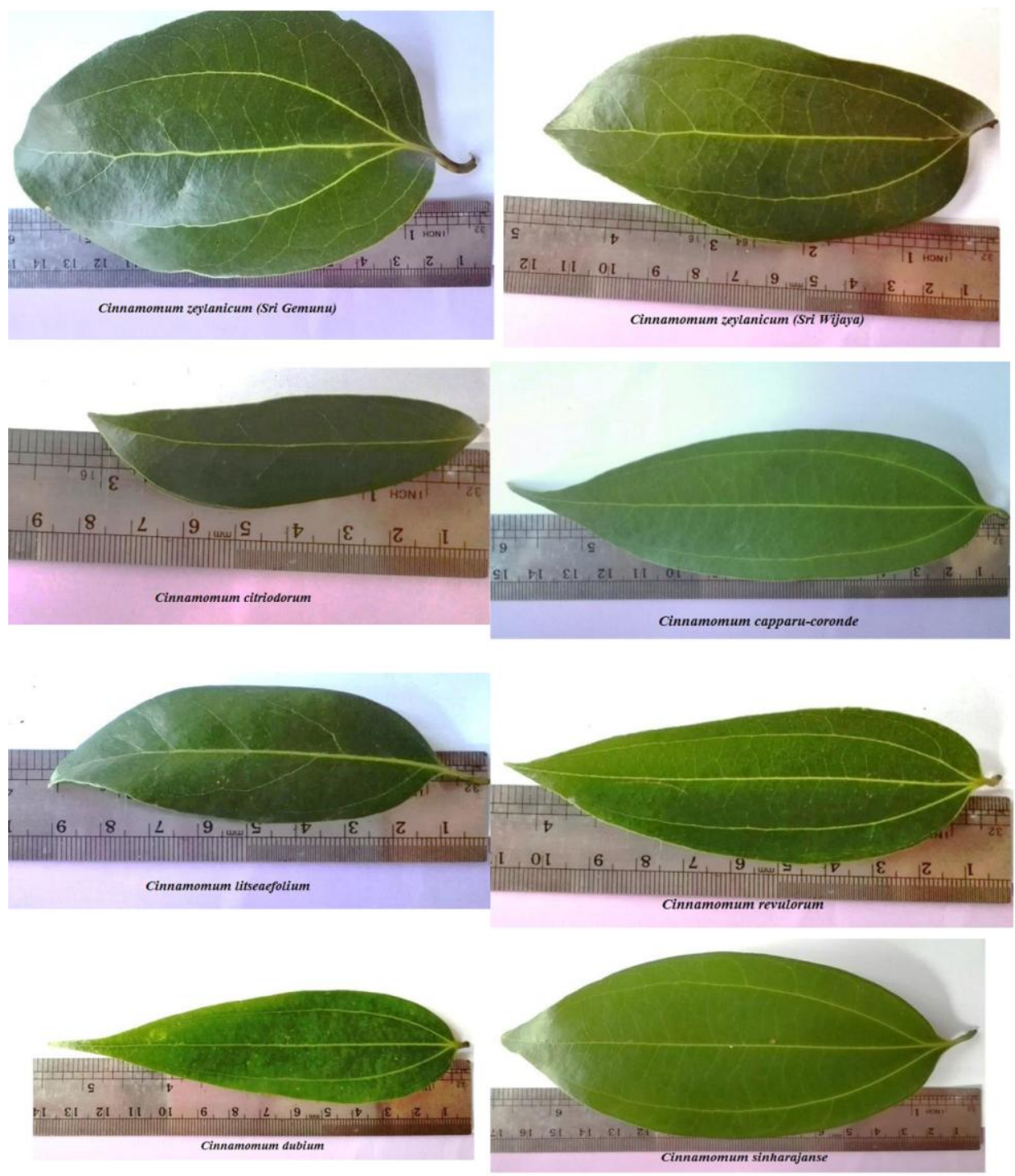

Figure 1: Leaf morphology of Cinnamon accessions used in the study 
Light brown barks were observed in $C$. zeylanicum (Sri Gemunu), C. zeylanicum (Sri Wijaya), C. capparu coronde, $C$. litseaefolium, $C$. revulorum and $C$. sinharajanse. Except $C$. capparu coronde and $C$. sinharajanse other tested species exhibited good peeling ability. Rough surface texture was observed in $C$. capparu coronde and wrinkled surface texture was observed in $C$. litseaefolium. All other test species have smooth surface texture. Aromatic odour was absent in $C$. litseaefolium, $C$. revulorum and $C$. sinharajanse and all others had the characteristic odours. C. zeylanicum (Sri Gemunu) and C. zeylanicum (Sri Wijaya) have slender branchlets. Light pink colour branchlets were observed in C. Capparu coronde. capparu-coronde and C. dubium was not significantly different from each other and ranged between 1.65-1.69 for leaves and 0.40-0.47 in bark. All species other than C. capparu-coronde and C. dubium have significantly different leaf oil contents. Highest mean leaf oil content was obtained from C. zeylanicum (Sri Gemunu) $(3.16 \%)$ and a considerably high leaf oil percentage $(2.96 \%)$ was obtained from C. zeylanicum (Sri Wijaya) and $C$. sinharajanse $(2.24 \%)$. The lowest mean leaf oil percentage was observed in $C$. revulorum $(0.39 \%)$ (Table 2$)$.

3.3 Quantitative analysis of leaf and bark oil content using GC-MS

The present study revealed that in addition to two cultivated cinnamon accessions, wild species such as $C$. capparu coronde and C. sinharajanse also

Table 3: Presence of Euginol, cinnamaldehyde and lonalool in leaf and bark oils of different cinnamon species tested.

\begin{tabular}{|c|c|c|c|c|c|c|}
\hline \multirow{3}{*}{ Species } & \multicolumn{6}{|c|}{ \% Similarity } \\
\hline & \multicolumn{2}{|c|}{ Eugenol } & \multicolumn{2}{|c|}{ Cinnzangldehyde } & \multicolumn{2}{|l|}{ Linalool } \\
\hline & Leaf & Bark & Leaf & Bark & Leaf & Bark \\
\hline C. zevlanicum (Sri Gemunu) & 24.329 & 5.173 & 0.608 & 17.781 & 1.149 & 15.988 \\
\hline C.zollanicumm (Sri JWjaya) & 28.285 & 6.128 & 0.177 & 19.830 & 5.253 & 18.751 \\
\hline C. citriodorum & - & - & - & 0.157 & 0.569 & 0.871 \\
\hline C. capparu coronde & 3.770 & 2.313 & 0.393 & 16.192 & 15.478 & 3.165 \\
\hline C litserafalinm. & 2.987 & 11.856 & 0.099 & 0.185 & 17.233 & 0.464 \\
\hline C. revularum & - & 0.867 & - & - & 0.707 & 0.323 \\
\hline C. dubium & - & - & - & - & 0.261 & - \\
\hline C. simhargjanse & 24352 & 2.474 & - & 12.877 & 26452 & 9783 \\
\hline
\end{tabular}

\subsection{Determination of leaf and bark oil yield}

Leaf and bark oil of $C$. zeylanicum (Sri Gemunu) and C. zeylanicum (Sri Wijaya) exhibited the characteristic smell while other species have different fragrances. Mean bark oil and leaf oil yield of $C$. contained a considerable amount of eugenol in leaf, while cinnamaldehyde is abundant in bark of $C$. sinharajanse (Table 3) confirming the ability of using those two species in crop improvement programs. 
3.4 Quantification of selected economically important phytochemicals present in leaf and bark oil

Highest eugenol content was found in leaves of $C$. zeylanicum (Sri Wijaya) and bark of $C$. litseaefolium. Eugenol is the major constituent in $C$. zeylanicum leaf oil accounting for about $80 \%$ of the total volatile oils (Senanayeke and Mubarak, 2001). Highest cinnamaldehyde content was found in leaves of $C$. capparu coronde which was almost 19 times high and in bark it was C. zeylanicum (Sri Wijaya) (Table 4). Cinnamaldehyde is the major constituent in C. zeylanicum bark oil, contributing about $70 \%$ of total oil (Senanayeke and Mubarak, 2001). Highest benzyl benzoate concentration of leaf oil and bark oil were found in $C$. litseaefolium (197036.18ppm) and $C$. zeylanicum (Sri Wijaya) (147151.1754 ppm) respectively (Table 4). two cultivated accessions and six wild species, those key characters can be used in morphological differentiation of studied species from each other.

C. sinharajanse had the highest mean leaf length, width and area (Sritharan, 1984) which is confirmed by the results of the present study. Natural environmental conditions where the species grown could largely affect the morphology and $C$. sinharajanse, which grows under high shade conditions, had larger leaves which are typical to plants growing under such conditions (Sritharan, 1984). In the present study although two cinnamon accessions and six wild cinnamon species grow under same environmental conditions, a significantly higher mean leaf length, width and area was found in C. sinharajanse indicating the larger size of the leaves are not merely due to environmental factors but also due to genetic characteristics.

Table 4: Eugenol, Cinnamaldehyde and Benzyl Benzoate concentration in leaf oil, bark oil $\left(10^{4} \mathrm{ppm}\right)$

\begin{tabular}{|c|c|c|c|c|c|c|}
\hline \multirow{2}{*}{ Species } & \multicolumn{2}{|c|}{ Eugenol } & \multicolumn{2}{|c|}{ Cinnamaldebyde } & \multicolumn{2}{|c|}{ Benzyl Benzoate } \\
\hline & Leaf & Bark & Leaf & Bark & Leaf & Bark \\
\hline C.zevilanicum (Sri Gemunu & 80.5 & 32.6 & 1.3 & 72.7 & 0.5 & 13.5 \\
\hline C. zevklanicum .Sri Wijoval & 86.4 & 35.9 & 0.9 & 75.4 & 2.1 & 14.7 \\
\hline C.citriadorum & - & - & - & 0.6 & 0.4 & 0.7 \\
\hline C. capparu corande & 31.1 & 0.6 & 19.3 & 58.0 & 16.4 & 2.3 \\
\hline C. litseraefolinm & 25.5 & 57.9 & 0.5 & 0.6 & 19.7 & 0.3 \\
\hline C. revularum & - & 5.4 & - & - & 0.7 & 0.2 \\
\hline C. dubium & - & - & - & - & 0.3 & - \\
\hline C. sinharajianse & 77.0 & 15.3 & - & 51.4 & 11.2 & 8.1 \\
\hline
\end{tabular}

\section{Discussion} 4.1 Determination and comparison of
morphological characters

Mean leaf length, width, fresh weight, petiole length and leaf area vary among
Studies on floral biology of cinnamon species by Senanayake and Mubarak (2001) reported that C. zeylanicum is predominantly an out crossing species and Sritharan (1984) suggested that this would in a way account for the high 
variability observed among the $C$. zeylanicum. However, present study revealed that, Sri Gemunu and Sri Wijaya being improved accessions of $C$. zeylanicum a less variability in leaf size, colour, fragrance etc. was exhibited, but showed variations only in shape and apex of the leaf.

The variations of leaf colour between tested cinnamon species are not a good parameter in distinguishing them separately. Although the leaf colour cannot be used, the flush colour could be considered as a useful character in differentiating studied Cinnamomum accessions to some extent.

The colour of the flower or the structure of the flower found to be less useful in distinguishing tested Cinnamomum species. However, seed parameters and bark characters could be used as a positive character in taxonomic identification of study species.

\subsection{Determination of leaf and bark oil yield}

As $C$. sinharajanse has a considerably high amount of both leaf and bark oil contents, this could be considered as a potential candidate for commercial purposes, however, a slight unpleasant odor in both leaf and bark oil of this species (Table1) should be taken into consideration and detail study on contents of oil is needed before using it for either crop improvement programs or in commercial purposes.

A high variability for oil percentage and its constituents have been reported (Sritharan, 1984) and the present study also revealed that although all tested species were grown under same environmental conditions, the content and constituents of cinnamon oil varies confirming that the microclimatic conditions have less effect on it, but the genetic makeup or other factors are mainly affecting the oil quality and quantity. Hence, identification, selection and propagation of superior varieties must be given a high priority in terms of improving the quality of cinnamon and oil content in bark and leaf.

\subsection{Quantitative analysis of leaf and bark oil content using GC-MS}

Cinnamon is mainly exported as quills and to certain extent as leaf and bark oil. The quality of quills greatly depends on the oil percentage and composition. The quality of bark and leaf oil depend on chemical constituents present, especially percentages of cinnamaldehyde and eugenol (Jayaprakasha et al., 2011). Cinnamon oils are demanded in the market in terms of the presence of eugenol in leaf oil and cinnamaldehyde in bark oil.

All parts of C. zeylanicum plant are capable of synthesizing the major volatiles found in the cinnamon oil. However, the leaf is a predominant site of eugenol and cinnamaldehyde synthesis (Senanayeke and Mubarak, 2001). Over 50 different compounds showed 95\% similarity in GC-MS analysis of bark oil samples and many of them are economically important compounds such as eugenol, benzyl benzoate, and camphene etc. Sritharan (1984) also reported the presence of Linalool in stembark, leaf and root-bark oils of all the Cinnamomum species which is confirmed by the present study. Among many of economically important volatile oils, linalool is a potential insecticide (Praveena and Sanjayan, 2011). Cinnamaldehyde, eugenol, and $\beta$ caryophyllene were identified as the most active antibacterial components in cinnamon bark and leaf oil (El-Baroty et al., 2010). Some of the better known mosquito repellents in cinnamon oil are geraniol (2,6-Octadien-1-ol, 3,7-dimethyl), citral (3,7-Dimethylocta-2,6-dienal), limonene (Cyclohexene, 4-methylene-1(1-methylethyl), pinenes, citronellol (6Octen-1-ol, 3,7-dimethyl-, ), citronellal (6Octenal, 3,7-dimethyl-, ) eugenol, and linalool (Moore et al., 2007). Present 
study revealed the presence of cinnamaldehyde and eugenol in two of the wild species, $C$. capparu coronde and C. sinharajanse in common and in small quantities in other tested wild species. Presence of those favorable characters indicate the possibilities of improvement of Ceylon cinnamon for these characters through hybridization programs.

\subsection{Quantification of selected economically important phytochemicals present in leaf and bark oil}

Present study revealed that in addition to two cultivated cinnamon accessions, some of the wild species namly- $C$. capparu coronde, $C$. litseaefolium and $C$. sinharajanse also contained eugenol in leaves (Table 4). Eugenol has great importance in pharmaceutical industry (Prakash and Gupta, 2005). The anticancer activity of eugenol has become an important research topic in recent years. Chemically synthesized anticancer drugs have toxic side effects and usually cause potential damage to normal cells (Xiaojun et al., 2014). Eugenol shows better application potential in the prevention and treatment of some cancers. Hussain et al., (2011) studied the effect of eugenol combined with gemcitabine on cervical carcinoma and found that the combination of eugenol and gemcitabine can inhibit cancer cell growth, even in low concentrations. Eugenol also has obvious killing or repellent action on worldwide agricultural insects, such as red flour beetle and citrus fruit fly males (Li et al., 2010).

Except $C$. revulorum and $C$. dubium, all other studied species contain cinnamaldehyde. Cinnamaldehyde is a constituent of essential oils known to exert anti-cancer, antifungal, cytotoxic mutagenic/anti-mutagenic and antiinflammatory effects (Rita et al., 2014). Babu et al., (2007) reported that cinnamaldehyde possesses hypoglycemic and hypolipidemic effects.

It was observed that benzyl benzoate in leaf oil of all species studied. Except in $C$. dubium benzyl benzoate was found in bark oil of all species studied. Benzyl benzoate is used as an acaricide, scabicide, and pediculicide in veterinary hospitals. It is also a repellent for chiggers, ticks, and mosquitoes. It is an effective and inexpensive topical treatment for human scabies. It has vasodilation and spasmolytic effects and is present in many asthma and whooping cough drugs (Hamid et al., 2015).

The clustering pattern of the selected two cultivated cinnamon accessions and six wild cinnamon species based on morphological, oil yield characteristics and chemical characters of bark and leaf oil is depicted in figure 2.

The analysis revealed that the studied species could be put into five clusters. $C$. sinharajanse was entirely different from the rest of the tested species and formed a separate cluster. Quantitative leaf parameters of $C$. sinharajanse (weight with petiole, length, width, length of petiole, leaf area). $C$. revulorum and $C$. litseaefolium have grouped with $C$. zeylanicum (Sri Wijaya) forming a cluster where $C$. litseaefolium and $C$. revulorum grouped together into a mini cluster. $C$. zeylanicum (Sri Gamunu) and C. capparu coronde have formed a one cluster and $C$. citriodorum and $C$. dubium have formed separate clusters individually. Based on the results obtained the following diagnostic key was developed in order to identify different Cinnamomum sp. tested (Figure 3). 


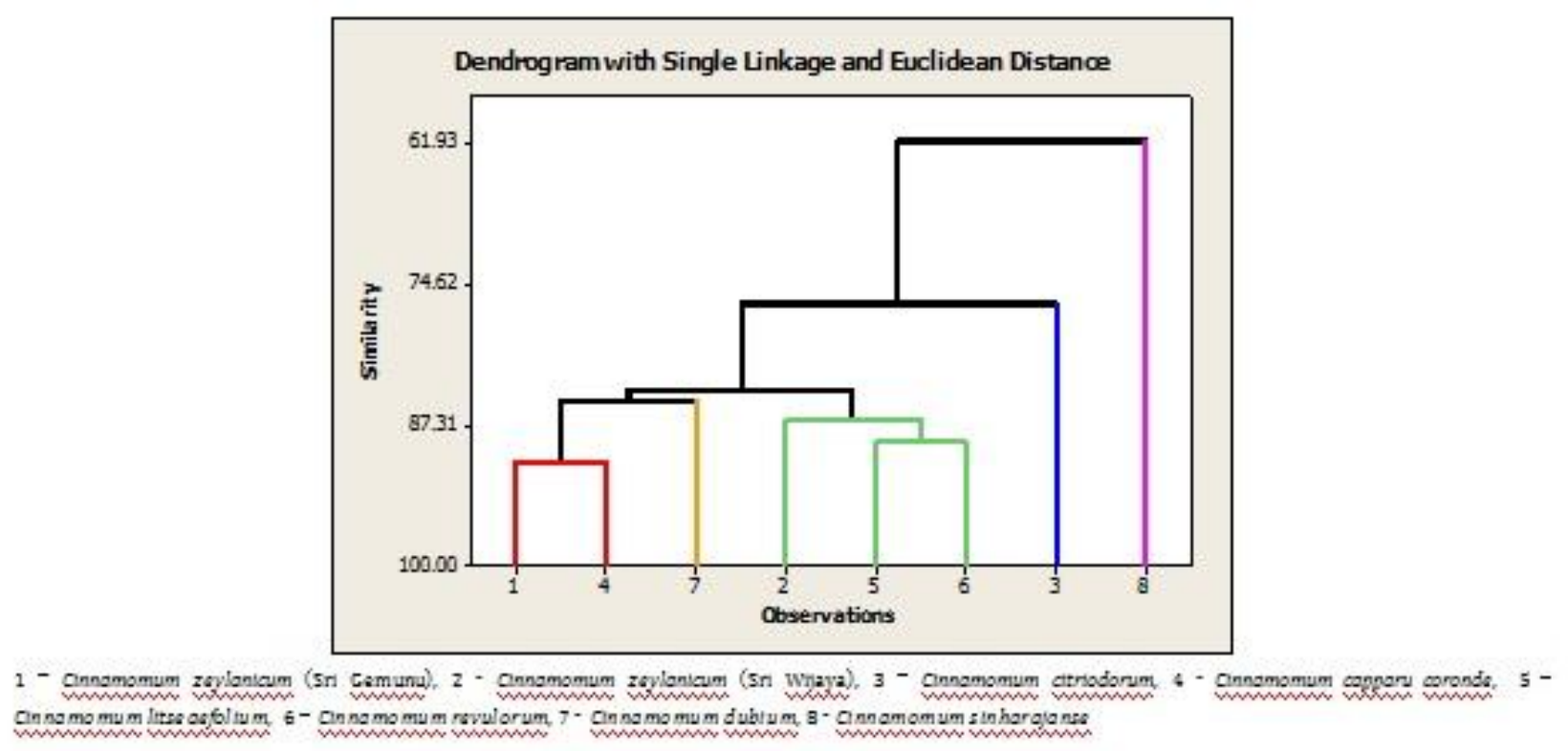

Figure 2: Dendrogram to illustrate the relationship between tested cinnamon species based on characteristics in morphology, oil yield and other phytochemicals present.

1. Pinnate venation present (prominent three veined venation absent) C. citriodorum

1. Prominent three veined venation present (midrib and two lateral veins prominent)

2. Contain eugenol in leaf oil

3. Not contain cinnamaldehyde in leaf oil C. sinharajaense

3. Contain cinnamaldehyde in leaf oil

4. Leaf lamina shape - oval

5. Leaf apex shape - acute. C. litseaefolium |

5. Leaf apex shape - ovate to eliptic. C. zeylanicum (Sri Gemunu)

4. Leaf lamina shape-lanceolate

5. Leaf apex shape - acute. C. zeylanicum (Sri Wiiaya)

5. Leaf apex shape - acuminate C. capparu-coronde

2. Not contain eugenol in leaf oil

3. Contain benzyl benzoate in bark oil

C. revulorum

3. Not contain benzyl benzoate in bark oil C.dubium

Figure 3: Key to species revised during the present study 


\section{Conclusions}

Mean leaf length, width, fresh weight, petiole length and leaf area vary among two cultivated accessions and six wild species indicating that those key characters can be used in morphological differentiation of studied species from each other. Significantly higher mean leaf length, width and area found in $C$. sinharajanse indicated that the larger size of the leaves are not merely due to environmental factors but also due to genetic characteristics.

The analysis revealed that the study species could be put into five clusters. $C$. sinharajanse was entirely different from the rest of the tested species and formed a separate cluster. Sri Gemunu and Sri Wijaya being improved accessions of $C$. zeylanicum exhibited a less variability in leaf size, colour, fragrance etc. but showed high variations in shape and apex of the leaf.

C. sinharajanse has a considerably high amount of both leaf and bark oil contents, this could be considered as a potential candidate for commercial purposes, however, a slight unpleasant odor in this species should be taken into consideration before using it for either crop improvement programs or in commercial purposes. Present study revealed the presence of cinnamaldehyde and eugenol in two of the wild species, $C$. capparu coronde and $C$. sinharajanse in common and in small quantities in other tested wild species. Thus those could be used in crop improvement programs in future.

\section{Acknowledgement}

Authors wish to acknowledge the support given by National Cinnamon Research and Training Center, Sri Lanka and University of Sri Jayawardenepura, Sri Lanka.

\section{References}

Babu, P.S., Prabuseenivasan S., Ignacimuthu, S. (2007) Cinnamaldehyde, a potential antidiabetic agent. Phytomedicine 14 (1): $15-22$.

El-Baroty, G. S., El-Baky, H. H. Abd., Farag, R. S., Saleh, M. A. (2010) Characterization of antioxidant and antimicrobial compounds of cinnamon and ginger essential oils. African Journal of Biochemistry Research 4 (6): 167-174.

Groot, R. S. D., Wilson, M. A., Boumans, R. M. J. (2002) A typology for the classification, description and valuation of ecosystem functions, goods and services. Ecological Economics 41: 393-408.

Gursale, A., Dighe, V., Parekh, G. (2010) Simultaneous Quantitative Determination of Cinnamaldehyde and Methyl Eugenol from Stem Bark of Cinnamomum zeylanicum Blume. using RP-HPLC. Journal of Chromatographic Science 48: 59-62.

Hamid, K.A., Ibrahim, S.I., Hashim, M.A., Salama, M. (2015) Formulation and Evaluation of Benzyl Benzoate Emulgel. Journal of Pharmacy and Biological Sciences 10: 06-09.

Hussain, A., Brahmbhatt, K., Priyani, A. (2011) Eugenol enhances the chemotherapeutic potential of gemcitabine and induce anticarcinogenic and antiinflammatory activity in human cervical cancer cells. Cancer Biother Radiopharm 26 (5): 519-27.

Jayaprakasha, G. K., Jagan Mohan Rao, L. (2011) Chemistry, Biogenesis, and Biological Activities of Cinnamomum zeylanicum. Critical Reviews in Food Science and Nutrition 51 (6): 547-562. 
Kostermans, A.J.G.H. (1995) Lauraceae. A Revised Handbook to the Flora of Ceylon. (eds. M.D. Dassanayaka, F. Fosberg \& W.D. Clayton), volume IX, pp. 112-129. Amerind Publishing Co. Pvt. Ltd., New Delhi, India.

Kulathilaka, P.S., Senarath, W.T.P.S.K. (2013) Determination of cytotoxicity and chemical identities in natural plants and callus cultures of Spilanthe spaniculata Wall. ex DC. International Journal of Herbal Medicine 1 (3): 135141.

Kush, G.S., Stebbins, G.L. (1961) Cytogenetic and Evolutionary studies in Secale. American Journal of Botany 48: 723-730.

Li, Y., Xiang, S., Zhu, W. (2010) Field experiment by using methyleugenol to trap and kill Dacus dorsalis (male). Pesticide Science and Administration 31 (6): 49-50.

Moore, SJ., Lenglet A., Hill N. (2007) Plant-based insect repellents, Chapter 14. In: Debboun M, Frances SP, Strickman D, editors.Insect repellents: principles, methods and uses. New York, USA: CRC Press, Taylor \& Francis Group; 2007.

Prakash, P., Gupta, N. (2005) Review article therapeutic uses of Ocimum sanctum Linn (Thulsi) with a note on eugenol and its pharmacological actions. Indian Journal Physiological Pharmacology 49 (2): 125-131.

Praveena, A., Sanjayan, K.P. (2011) Inhibition of acetylcholinesterase in three insects of economic importance by linalool, a monoterpene phytochemical. Insect Pest Management 1: 340-345.

Rita, D.C.D.S., Luciana, N.A., Rafael B.O., Damião P.S. (2014) A Review on Anti- Inflammatory activity of Phenylpropanoids found in essential oils. Molecules 19: 1459-1480.
Saumyasiri, M.M.K.G., Yakandawala, D.M.D., Samaraweera, P., Wijesinghe, K.G.G. (2006) Phylogenetic Relationships of Cinnamomum species in Sri Lanka. Proceedings of the $11^{\text {th }}$ Peradeniya Universaity Research Sessions, 30 November, p.134.

Senanayake, U.M., Mubarak, A.M. (2001) A comparison of essential oil constituents of bark, leaf, root and fruit of cinnamon (Cinnamomum zeylanicum Blume.) grown in Sri Lanka. Journal of the National Science Foundation of Sri Lanka 29 (3\&4): 147-153.

Sritharan, R. (1984) The study of genus Cinnamomum. M.Phil.Thesis, Post Graduate Institute of Agriculture, University of Peradeniya, Sri Lanka.

Wijesinghe, K.G.G., Samaraweera, D.N., Jayasinghe, D., Gunaratna, G.G. (2004) Development of Cinnamon (Cinnamomum verum Presl) selection for higher yields with better quality characteristics. CARP Competitive Contract Research Grants Programme (eds. H.P.M. Gunasena, M.R. Dhanapala \& T.U. Tilekawardana), pp 3-10. Sri Lanka Council for Agricultural Research Policy, Wijerama Mawatha, Colombo 07.

Xiaojun, K., Xiwang, L., Jianyong ,L., Yajun, Y. (2014) Advances in Pharmacological Research of Eugenol. Current opinion complement alternative medicine 1 (1): 8-11. 